\title{
Interações de planorbídeos vetores da esquistossomose mansoni e o problema da expansão de endemia na região amazônica*.
}

\section{The interations of planorbic vectors of Schistosoma mansoni and their relations with the expansion of the endemic disease in the Amazon region.}

\author{
Roxana Valadāo**, Roberto Milward-de-Andrade ${ }^{* \star}$
}

\begin{abstract}
"No tocante a sua vegetação, a planta convém com a besta bruta e, por sentimento, o animal brutal com o homem, que se conforma ao resto dos astros por sua inteligência; essa ligação procede tão apropriadamente que parece uma corda estendida desde a primeira causa até as coisas baixas e ínfimas, por uma ligação recíproca e contínua; de sorte que a virtude superior, expandindo seus raios, chegará a tal ponto que, se the tocarmos uma extremidade tremerá e fará mover o resto" (C.G. Porta. Magie Naturelle, 1650).
\end{abstract}

\begin{abstract}
VALADĀO, R. \& MILWARD-DE-ANDRADE, R. Interação de planorbídeos vetores da esquistossomose mansoni e o problema da expansão de endemia na região armazônica. Rev. Sáude públ., S. Paulo, 25: 353-8, 1991. A hipótese da superioridade competitiva é discutida levando-se em conta duas questões a ela relacionadas: uma referente à complexidade dos fenômenos envolvidos na interação e distribuição das populações de planorbídeos e, outra, ligada à interação que se estabelece entre os caramujos distribuídos numa dada região e o homem ou animal infestado, aí presente. Destaca-se a distribuição de planorbídeos na Amazônia, e o problema da endemia na região é levantado.
\end{abstract}

Descritores: Biomphalaria. Distribuição espacial. Esquistossomose mansônica, epidemiologia.

\section{Introdução}

A esquistossomose mansoni constitui um dos mais graves e complexos problemas de saúde pública em nosso território. Apresenta uma nítida tendência à expansão que decorre, de modo particular, das migrações humanas a partir de áreas endêmicas, como no caso de sua introdução, relativamente recente, na Região Amazônica ${ }^{1626,27,32,34}$. A expansão da endemia, sendo condicionada pelos fluxos migratórios, ocorre quando estes possibilitam a reunião de populações humanas infestadas e planorbíneos susceptíveis ao parasita9.

Assim, uma avaliação dos fatores envolvidos no estabelecimento de novos focos da doença deve levar em conta não apenas a existência de hospedeiros intermediários e a distribuição de suas populações numa dada região, mas também, as condições sócio-econômicas das règiōes afetadas. A consideração destes aspectos, embora não seja importante em estudos de outra natureza, torna-se imprescindível quando se trata de fundamentar uma ação de controle epidemiológico da doença.

* Financiado parcialmente pelo Conselho Nacional de Desenvolvimento Científico e Tecnológico (CNPq) Apresentado ao I Simpósio Internacional de Estudos Ambientais em Florestas Tropicais Úmidas, Manaus, 1990.

** Departamento de Biologia do Instituto Oswaldo Cruz da Fundação Instituto Oswaldo Cruz (FIOCRUZ), Rio de Janeiro, RJ - Brasil.

Separatas/Reprints: R. Valadão - Av. Rio Branco, 2929/ 1302 - 36100 - Juiz de Fora, MG - Brasil.
Dentro desta perspectiva, avalia-se, aqui, a hipótese da superioridade competitiva, formulada por alguns autores a partir de dados obtidos em interações populacionais de Biomphalaria glabrata e $B$. straminea, onde a primeira foi substituída pela segunda. Isto, porque a condição de superioridade competitiva, aliada a resultados de laboratório, que revelam a menor susceptibilidade de $B$. straminea ao Schistosoma mansoni, poderia justificar a ação de controle pela substituição de uma espécie ( $B$. glabrata) por outra (B. straminea).

Na presente avaliação, duas questões são consideradas: a primeira diz respeito à complexidade dos fenômenos envolvidos na interação e distribuição populacional dos planorbídeos e, a segunda, referese à relação que se estabelece entre os caramujos distribuídos numa dada região e as fontes de infecção aí presentes. A distribuição dos planorbídeos vetores na Região Amazônica é destacada, uma vez que se questiona, neste estudo, a situação da endemia na região.

\section{A complexidade dos fenômenos envolvidos na distribuição dos planorbídeos}

A hipótese da superioridade competitiva e a distribuição das espécies

B. glabrata, B. straminea e B. tenagophila são reconhecidas como as três principais espécies hospedeiras intermediárias do $S$. mansoni em nosso meio. Enquanto $B$. tenagophila é mais freqüente na Região Sul, tendo importância epidemiológica aparentemente restrita, $B$. straminea exibe uma ampla distribuição, ainda que seu papel mais importante 
na transmissão da doença refira-se à Região Nordeste, onde, em algumas áreas é o único transmissor. Por sua vez, $\boldsymbol{B}$. glabrata revela-se como a principal responsável pela manutenção da endemia em extensas áreas de nosso território, apresentando uma distribuição que abrange desde a Região Nordeste à Região Sudeste e inclui localidades das Regiões Norte, Centro-Oeste e Sul ${ }^{9,32,33,34}$.

Embora existam áreas de superposição na distribuição geográfica dessas espécies, apenas raramente são encontradas em um mesmo criadouro ${ }^{3,25,30,32}$. Esta condição de co-existência inter-específica é citada para $B$. glabrata e $B$. straminea, além de $B$. glabrata e $B$. tenagophila, em Minas Gerais ${ }^{29.32}$ e, para $B$. tenagophila e $B$. straminea, em São Paulo ${ }^{\prime 1}$. Registros de um caso de interação de $B$. glabrata de Aracaju (SE) introduzida na área de Manguinhos (RJ), num criadouro de $B$. tenagophila, mostram que não houve erradicação da espécie autóctone, mesmo depois de decorridos 54 anos da introdução da espécie alienígena ${ }^{37}$.

Paralelamente, a existência de competição interespecífica com deslocamento e substituição de uma espécie por outra, tem sido mencionada por diversos autores. O registro de um caso de substituição de populações de $B$. glabrata nativa, em Belo Horizonte (MG), por B. tenagophila oriunda de São Paulo, num tempo relativamente curto ${ }^{37}$, ilustra este fato. Outro exemplo significativo, é o da periferia de Recife (PE), onde houve substituição de populações autóctones de $B$. glabrata por $B$. straminea introduzida ${ }^{4}$, sendo que a distribuição atual das duas espécies parece ser o resultado de um processo de deslocamento ocorrido no período entre 1957 e $1978^{5}$. Na Martinica, B. glabrata é mencionada, hoje, como uma espécie rara em decorrência de sua possível substituição natural por $B$. straminea ${ }^{12}$. Fenômeno inverso, ao que parece, vem ocorrendo em Belém (PA), com o surgimento de $B$. glabrata em locais, supostamente ocupados apenas por $B$. straminea $a^{35,36}$.

Dados obtidos a partir de interações populacionais de $B$. glabrata e $B$. straminea, onde a primeira foi substituída pela segunda, levaram alguns autores a formular a hipótese da superioridade competitiva de uma espécie sobre outra ${ }^{5,12.17}$. Tal superioridade estaria fundamentada em algumas características encontradas para $B$. straminea, tais como a maior resistência à dessecação e à infestação por $S$. mansoni $i^{4.7}$, a maior vagilidade e capacidade de dispersão ${ }^{6.17}$ desta espécie, ou ainda a sua maior fecundidade em relação à $B$. glabrata ${ }^{17}$.

Entretanto, os aspectos anteriormente relatados acerca das interações de $B$. glabrata, $B$. straminea e $B$. tenagophila sugerem, antes, que a superioridade competitiva de uma espécie sobre outra pode ter valor relativo e ser dependente da combinação momentânea de diversos fatores: as vantagens atribuí- das à espécie dominante revelam-se insuficientes para explicar a distribuição geográfica das espécies, quando confrontadas com as diferentes formas possíveis de interações populacionais (deslocamento, superposição, co-existência), mostradas pelas pesquisas de campo.

A hipótese da superioridade competitiva e a dificuldade de se estabelecer o conjunto de elementos pertencentes a este conceito

As características físicas e químicas do meio ambiente constituem fatores reconhecidamente capazes de influenciar na distribuição dos moluscos planorbídeos ${ }^{2.15,18,19,32}$. No entanto, as interações recíprocas existentes entre as espécies de caramujo e o meio são, até agora, insuficientemente conhecidas, permanecendo obscuros os fatores que remetem a uma padrão de exclusão competitiva ("regionalização" relativa, deslocamento e substituição de espécies) e os mecanismos adaptativos que possibilitam a co-existência das espécies num mesmo biótopo, em condições naturais.

Em pesquisas de campo e estudos experimentais sobre populações, a utilização das taxas de crescimento individual, de oviposição, de mortalidade e outras, reveste-se de valor heurístico, uma vez que, estas, representam avaliações numéricas da competição que se estabelece entre os organismos. Tais são os parâmetros de algumas investigações envolvendo diferentes espécies de moluscos, onde as alterações detectadas no seu desenvolvimento individual e populacional foram atribuídas à competição inter ou co-específica e relacionadas, basicamente, à variação nas densidades dos grupos em interação e nas concentrações de algumas substâncias diluídas no meio ${ }^{1,20,21,22,23,24.25,40}$.

Nesta perspectiva, as densidades dos grupos em interação constituem um fator que pode levar ao incremento das taxas de crescimento individual, de sobrevivência e de oviposição, quando dentro de certos limites ("efeito de grupo") ou à redução das mesmas, se acima de determinados níveis ("efeito de massa"). Neste último caso, ao que tudo indica, o que ocorre é uma ação difusa, de "stress", sobre os indivíduos. A informação detectada pelo animal, sobre as possibilidades de sobrevivência e reprodução no ambiente, é inespecífica, sendo determinada pela depleção de substâncias necessárias ao metabolismo e pela concentração elevada de produtos de excreção dos indivíduos aí presentes. A inespecificidade desta ação prende-se, também, ao fato de que as variações nas concentrações das referidas substâncias traduzirse-ão em condições ambientais desfavoráveis para quaisquer das espécies em interação, ainda que a faixa de tolerabilidade varie para as diferentes espécies.

É possível, além disto, distinguir na interação dos grupos de indivíduos, um tipo de informação que é 
mais específica, no sentido de que as substâncias produzidas por um deles atua sobre o outro, mesmo na ausência de condições desfavoráveis do meio, podendo ou não, atuar sobre o grupo que as produz, sob certas circunstâncias ${ }^{8,13}$. A este respeito, uma pesquisa realizada com populações de Fossaria cubensis e B. glabrata $^{14}$, onde uma substância inibidora ("IS") foi isolada a partir de culturas de $F$. cubensis, mostrou que esta levava à inibição do crescimento e reprodução de $B$. glabrata, por um mecanismo denominado de "cross species effect". -

Os resultados das pesquisas aqui mencionadas e as considerações por eles suscitadas, permitem admitir que os fatores implicados na interação e distribuição das populações de moluscos se interrelacionam e imbricam numa complexa rede de fenômenos e de possibilidades. Esta complexidade de ocorrências associada à interação populacional explicaria, ao menos parcialmente, as dificuldades para uma compreensão clara dos mecanismos e componentes envolvidos na exclusão competitiva ou na co-existência inter-específica.

\section{Relação planorbideo susceptivel-esquistos- somose e o problema da endemia na Região Amazônica}

\section{A substituição de espécies e o ciclo vital do para- sita}

Uma avaliação da extensão e do potencial expansivo da esquistossomose, deve incluir, além dos aspectos relacionados à distribuição dos caramujos, aqueles relativos à distribuição das fontes de infecção'. Esta circunstância decorre da complexidade ecológica, característica da esquistossomose, dada por suas relações com o hospedeiro intermediário e o definitivo. Tais relações constituem-se em condições bio-ecológicas particulares que favorecem o fechamento do ciclo vital do parasita e, assim, propiciam a manutenção e disseminação da doença.

Resumidamente, as referidas condições podem ser descritas da seguinte maneira ${ }^{38}$ : a existência de fontes de infecção (homem ou animal parasitado); a presença de, pelo menos, uma das espécies vetoras do gênero Biomphalaria; a existência de coleções de água doce adequadas à vida do molusco e às fases de vida livre do parasita; os hábitos da população (condições econômicas, de moradia, de saneamento, e outras).

Alguns trabalhos, tocando esta questão da interdependência entre o hospedeiro intermediário e o definitivo, chamam a atenção para o fato de que o avanço da esquistossomose, no país, acompanha as correntes de migração humana, sendo condicionado pela presença do molusco hospedeiro intermediário $^{31.32,33.34,35}$.

Assim, a penetração da parasitose na Amazônia é apontada como resultado da imigração de nordesti- nos, atraídos pelas plantações de Hevea brasiliensis que produziam o latex ${ }^{26}$. Do mesmo modo que o surgimento da doença, no Município de Fordlândia (PA), é atribuído aos imigrantes, o seu declínio posterior é imputado à dispersão dos trabalhadores empregados pela Ford Motor Company, que ocorreu depois do fracasso desta empresa ${ }^{36}$. Num estudo a respeito da epidemiologia da esquistossomose em São Paulo9, demonstra-se que o deslocamento de áreas endêmicas associa-se ao processo de desenvolvimento do país que, em determinadas etapas, produziu importantes migrações de mão de obra, em busca de trabalho. Tendo ocorrido a partir de áreas endêmicas, estas migrações propiciaram as condições para a reunião das populações humanas infestadas e os planorbídeos susceptíveis, em virtude da não implantação prévia de uma infra-estrutura de saneamento nas regiões ocupadas.

Se o parasita realiza seu ciclo vital em um conjunto de etapas que tocam, fundamentalmente, ao homem (ou animal) parasitado e ao molusco susceptível (os quais, por sua vez, interagem reciprocamente, através dele), um projeto de controle epidemiológico deve abranger estas etapas. Mas não apenas isto. $\mathrm{O}$ modo como se opera nestas etapas é também importante, dada a condição de reciprocidade acima referida.

No que diz respeito ao caramujo, a perspectiva da relação de condicionamento recíproco altera os fundamentos de uma ação pautada na substituição de espécies, ao introduzir um elemento ligado, não ao caramujo, mas, sim, ao homem (ou animal) parasitado. Esta consideração é válida, não apenas para o grupo dos planorbídeos, mas também, para os demais grupos de moluscos competidores, eventuais transmissores de outras parasitoses.

Quanto a este ítem, são sugestivos os relatos transcritos, sucintamente, a seguir.

No Estado do Pará, seis casos positivos foram registrados, no ano de 1934, em algumas localidades $^{36}$, tendo sido ressaltado que estas amostras poderiam ser de imigrantes infectados em outras regiões. Mais tarde ${ }^{28}$ foram encontradas amostras positivas em material de viscerotomia proveniente de diversos municípios deste mesmo Estado, embora não se tenha esclarecido se, estes, constituiam focos endêmicos. Finalmente, em 1949, foram feitos registros de casos autóctones, sendo que estes primeiros focos de infecção eram mantidos pela $B$. straminea ${ }^{26,36}$.

Este quadro, atualmente, parece modificar-se em função do achado de $B$. glabrata associada à esquistossomose em algumas localidades ${ }^{35}$. Chamamos a atenção para o fato porque, de um lado, o achado de $B$. 'glabrata, em locais anteriormente ocupados por $B$. straminea, constitui uma evidência a mais das interações cambiantes e sutis que se 
estabelecem entre o caramujo e o ambiente. De outro lado, este dado é relevante por insertar-se na problemática da relação planorbídeo-parasitose, na medida em que o molusco surge associado à esfuistossomose em áreas já consideradas endêmicas vara a doença.

A substituição de espécies e a susceptibilidade ao parasita

Um outro dado, ainda no enfoque do condicionamento recíproco, refere-se à positividade dos índices endêmicos de esquistossomose, associada à susceptibilidade do caramujo. Neste caso, a questão seria: é possível encontrar uma alta prevalência de esquistossomose relacionada a planorbídeos pouco susceptíveis?

A propósito desta pergunta, são exemplares os municípios de Gameleira, Vicência, Escada e Ribeirão, no Estado de Pernambuco, que apresentavam, na década passada, índices de positividade para a esquistossomose, de $70 \%$ a $90 \%$. Também podem ser lembrados os focos isolados da doença assinalados para os municípios de Pacoti, Quixadá, Redenção e Juazeiro do Norte, no Estado do Cearáa ${ }^{39}$.

Para todas estas localidades, Paraense ${ }^{32}$ menciona, exclusivamente, a presença de $B$. straminea, reconhecida como pouco susceptível ao $S$. mansoni.

São, ainda, significativos os registros sobre $B$. amazonica. Um levantamento da distribuição de planorbídeos, na Região Norte ${ }^{34}$, revela a presença desta espécie em extensos segmentos ao longo da bacia hidrográfica do rio Amazonas. Ao lado disto, dados experimentais ${ }^{10}$ indicam que $B$. amazonica é susceptível ao $S$. mansoni (taxas de infecção variando entre $48 \%$ e $73 \%$, de acordo com a cepa do parasita utilizada), sendo considerada um vetor potencial da esquistossomose, mais susceptível que $B$. straminea.

Entretanto, até o momento, não se tem notícia da ocorrência de focos endêmicos associados a este caramujo.

É possível supor, a partir destas situações, que, mesmo um tipo de ação cujo objetivo específico seja o controle do caramujo, deve ter em perspectiva a adesão, deste, ao seu ciclo vital próprio, que o coloca numa relação de condicionamento recíproco com o homem (ou animal) parasitado - ambos sendo necessários para a (re)introdução do parasita na cadeia epidemiológica da esquistossomose.

Distribuição dos planorbídeos na bacia do rio Amazonas

A Amazônia Legal compreende uma área de $4.978 .247 \mathrm{~km}^{2}$ ou $58,5 \%$ do território nacional, abrangendo as seguintes unidades da Federação: Amazonas, Pará, Acre, Rondônia, Amapá e Roraima. No caso da bacia do rio Amazonas, incluem-se
Maranhão, Mato Grosso, Mato Grosso do Sul, Goiás e Distrito Federal.

A fauna de planorbídeos, na bacia hidrográfica em questão, compõe-se, pelo menos, 14 espécies, 8 das quais pertencentes ao gênero Biomphalaria, sobressaindo-se $B$. glabrata como transmissora comprovada, $B$. straminea como vetora provável do foco, ora extinto, de Fordlândia (PA), $B$. peregrina e $B$. amazonica, como vetoras potenciais ${ }^{26.34 .35}$.

Observa-se que ainda é modesto o conhecimento da distribuição geográfica dos moluscos de interesse médico, ou das espécies comprovadamente susceptíveis ao $S$. mansoni, na região.

Os dados que demonstram esta circunstância são apresentados na Tabela e referem-se ao número de localidades nas quais as espécies susceptíveis foram encontradas.

Tabela. Localidades onde foram encontradas espécies susceptiveis ao Schistosoma mansoni.

\begin{tabular}{|c|c|c|c|c|}
\hline $\begin{array}{l}\text { Unidade } \\
\text { Federativa }\end{array}$ & $\begin{array}{c}B . \\
\text { glabrata }\end{array}$ & $\begin{array}{c}B . \\
\text { straminea }\end{array}$ & $\begin{array}{c}B . \\
\text { peregrina }\end{array}$ & $\begin{array}{c}B . \\
\text { amazonica }\end{array}$ \\
\hline $\begin{array}{l}\text { Amazonas } \\
(16)\end{array}$ & - & - & - & 10 \\
\hline $\begin{array}{l}\text { Pará } \\
(20)\end{array}$ & 3 & 17 & - & - \\
\hline $\begin{array}{l}\text { Acre } \\
(7)\end{array}$ & - & 3 & - & 2 \\
\hline $\begin{array}{l}\text { Rondônia } \\
\text { (3) }\end{array}$ & - & - & - & 2 \\
\hline $\begin{array}{c}\text { Amapá } \\
\text { (1) }\end{array}$ & - & - & - & - \\
\hline $\begin{array}{c}\text { Roraima } \\
\text { (1) }\end{array}$ & - & 1 & - & - \\
\hline $\begin{array}{l}\text { Maranhão } \\
\text { (24) }\end{array}$ & 8 & 15 & - & - \\
\hline $\begin{array}{l}\text { Mato Grosso } \\
\text { (21) }\end{array}$ & - & 1 & - & - \\
\hline $\begin{array}{l}\text { M.G. do Sul } \\
\text { (12) }\end{array}$ & - & 1 & 2 & - \\
\hline $\begin{array}{l}\text { Gọoiás } \\
\text { (24) }\end{array}$ & 1 & 11 & 1 & - \\
\hline $\begin{array}{l}\text { Dist.Federal } \\
\text { (1) }\end{array}$ & 1 & 1 & 1 & - \\
\hline
\end{tabular}

() Entre parênteses encontra-se o número de localidades levantadas em cada Unidade Federativa considerada.

Os dados da Tabela revelam a necessidade de conhecimentos mais amplos sobre a distribuição espacial dos planorbídeos. Isto é importante, tendo em vista os riscos de expansão da endemia nessa região, onde a abertura de estradas, os empreendimentos agro-pecuários, a instalação de usinas hidrelétricas e empresas de mineração funcionam como polos de atração para a mão de obra em busca de uma melhor inserção no mercado de trabalho. 


\section{Agradecimento}

À Lygia R. Corrêa, do Departamento de Malacologia/Instituto Oswaldo Cruz/Fundação Instituto Oswaldo Cruz, pelas valiosas críticas e sugestões.

VALADÃO, R. \& MILWARD-DE-ANDRADE, R. [The interactions of planorbic vectors of Schistosoma mansoni and their relations with the expansion of the endemic disease in the Amazon region]. Rev. Saúde públ., S. Paulo, 25: $353-8,1991$. The hypothesis of competitive superiority is discussed in the light, first, of the complexity of the phenomena involved in the interaction and distribution of planorbid populations and, secondly, of the relation that is established between the snails of any given region and the infected human or animal host. Special attention is given to the distribution of planorbids in the Amazon, and the endemic problem of the region is dealt with.

Keywords: Biomphalaria. Residence characteristics. Schistosomiasis mansoni, epidemiology.

\section{Referências Bibliográficas}

1. AHMED, M.D.; UPATHAM, E.S.; BROKELMAN, W.Y.; VIYANANT, V. Populations responses of the snail Bulinus (Physopsis) abyssinicus to differing inicial social and crowding conditions. Malacol. Rev'., 19: 83-9, 1986.

2. APPLETON, C.C. Review literature on abiotic factors influencing the distribution and life cycles of bilharziasis intermediate host snails. Malacol. Rev., 11: 1-25, 1978.

3. BARBOSA, F.S. \& OLIVIER, L. Studjes on the snail vectors of bilharziasis mansoni in the North-Eastern Brazil. Bull Wld Hlth Org., 18: 895-908, 1958.

4. BARBOSA, F.S. Possible competitive displacement and evidence of hybridization between two Brazilian species of planorbid snails. Malacologia. 14: 401-8. 1973.

5. BARBOSA, F.S.; COSTA, D.P.P. da; ARRUDA, F. New field observations on the competitive displacement between two species of planorbid snails inhabiting NorthEastern Brazil. Mem. Inst. Oswaldo Cruz, Rio de Janeiro. 76: $361-6,1981$.

6. BARBOSA, F.S.; COSTA, D.P.P. da; ARRUDA, F Competitive inter-actions between species of freshwater snails. l. Laboratory studies: Ib. Comparative studies of the dispersal and vagility capabilities of Biomphalaria glabrata and B. straminea. Mem. Inst. Oswaldo Cruz, Rio de Janeiro, 79: 163-7, 1984.

7. BARBOSA, F.S. Competitive displacement of Biomphalaria glabrata by $B$. straminea. Mem. Inst. Oswaldo Cruz, Rio de Janeiro, 82: 139-41, 1987

8. BOUSFIELD, J.D.; TAITI, A.I.; THOMAS, J.D.: TOWNERJONES, D. Behavioral studies on the nature of stimuli responsible for triggering mucus trail tracking by Biomphalaria glabrata. Malacol. Rev., 14: 49-64, 1981.

9. CHIEFFI, P.P. \& WALDMAN, E.A. Aspectos particulares do comportamento epidemiológico da Esquistossomose Mansônica no Estado de São Paulo, Brasil. Cad. Saúde públ., Rio de Janeiro, 4: 257-75, 1988.

10. CORREA, L.R. \& PARAENSE, W.L. Susceptibilily of Biomphalaria amazonica with two strains of Schistosoma mansoni. Rev. Inst. Med. trop. S. Paulo, 13: 387-90, 1971.

11. CORRÊA, R. de R.; MURGEL, J.M. de T.; PIZA, J. de T.; RAMOS A. da S.; DIAS, L.C. de S.; MORAIS. L.V.C.; ROSARIO, F.F. do. Dispersão de Biomphalaria straminea, hospedeira intermediária do Schistosoma mansoni, através da distribuição de peixes. Rev. Saúde públ., S. Paulo, 4: 117-27, 1970.
12. GUYARD, A. \& POINTER, J.P. Faune malacologique dulçaquicole et vecteurs de la Schistosome intestinale en Martinique. Ann. Parasit., Paris, 2 (54): 193-205, 1979.

13. HAMILTON, P.V. The use of mucous trails in gastropod orientation studies. Malacol. Rev., 10: 73-6, 1977.

14. LEVY, M.G.; TUNIS, M.; ISSEROFF, H. Population control in snails by natural inhabitors. Nature, 241: 65-6, 1973.

15. MALEK, E.A. Snail hosts of schistosomiasis and other snuiltransmitted diseases in tropical America: a manual. Washington, D.C., Pan American Health Organization, 1985.

16. MAROJA, R.C. Incidência da esquistossomose em Fordlândia, município de Itaituba, Estado do Pará. Rev. Serv. esp. Saúde públ., 6: 211-8, 1953.

17. MICHELSON, E.H. \& DUBOIS, L. Competitive interaction between two snail hosts of Schistosoma mansoni: laboratory studies on Biomphalaria glabrata and $B$. straminea. Rev. Inst. Med. trop. S. Paulo, 21: 246-53, 1979.

18. MILWARD-DE-ANDRADE, R.; SANTOS, I.N.; OLIVEIRA, R. Contribuição para o conhecimento dos criadouros de planorbídeos, na área do Distrito Federal. 1. Variação de diferentes fatores químicos de suas águas. Rev. bras, Malar., 7: 103-30, 1955.

19. MILWARD-DE-ANDRADE, R. Ecology of Australorbis glabratus in Belo Horizonte, Brazil. I. Annual fluctuation in population density and in infecton indices by Schistosoma mansoni (Pulmonata: Planorbidae). Rev. bras. Malar., 14: 261-89, 1962.

20. MILWARD-DE-ANDRADE, R. \& CARVALHO, O.S. Alimentação e fecundidade de planorbídeos criados em laboratório. 1. Biomphalaria tenagophila (Orbigny, 1835) (Pulmonata: Planorbidae). Rev. bras. Biol., 32: 225-33, 1972.

21. MILWARD-DE-ANDRADE, R.; CARVALHO, O.S.; ALVES, M.P.P. Alimentação e fecundidade de planorbídeos criados em laboratório. II. Biomphataria straminea (Dunker, 1848). Rev. bras. Biol, 33: 119-26, 1973.

22. MILWARD-DE-ANDRADE, R.: CARVALHO, O.S.: MENEZES, W.T. Alimentação e fecundidade de planorbídeos criados em laboratório. III. Biomphalaria glabruta. Rev. bras. Malar., 26/27: 109-29, $1974 / 75$.

23. MILWARD-DE-ANDRADE, R. Competição entre Helisama duryi (Wetherby, 1879) e Biomphalaria glabrata (Say, 1818 ), em condiçōes de laboratório (Pulmonata, Planorbidac). Rev. hras. Biol., 38: 787-800, 1978.

24. MILWARD-DE-ANDRADE, R. Natalidade comparada de Helisoma duryi e Biomphalaria glabrata, em laboratório (Mollusca. Planorbidae). Rev. bras. Malar., 31: 35-58, 1979.

25. MILWARD-DE-ANDRADE, R. \& CARVALHO, O. dos S. Colonização de Pomucea haustrum (Reeve, 1866) em localidade com esquistossomose mansoni: Baldim, MG (Brasil) (Prosobranchia, Palidae). Rev. Saúde púhl., S. Paulo, 13: 92-107, 1979.

26. MILWARD-DE-ANDRADE, R. Diseases related to the aquatic environment in the Amazon. In: Sioli, H., ed. The Amazon: limnology and landscape ecology of $a$ mighty river and ist basin. Netherlands, Dr. W. J. Publ., 1984. p. 477-99.

27. MORAES, M.A.P. A esquistossomose na Amazônia, Brasil. Rev. Univ. Fed. Pará. 2: 197-219, 1972.

28. PARA, M. Dado's estatísticos de viscerotomia sobre doença e condições mórbidas do homem no Brasil. I. Schistosomose mansônica no período de 1937-1946. Mem. Inst. Oswaldo Cruz, Rio de Janeiro, 47: 443-519, 1949.

29. PARAENSE, W.L. \& DESLANDES, N. Studies on Australorbis centimentralis. II. Biospecificcharacterization. III. Generic status. Rev. bras. Biol., 15: 293-307, 1965.

30. PARAENSE, W.L. \& DESLANDES, N. A "nova sistemática" aplicada aos planorbídeos brasileiros. Rev. Serv. esp. Saúde públ., 8: 491-510, 1956. 
31. PARAENSE, W.L. Planorbídeos hospedeiros intermediários do Schistosoma mansoni. In: Cunha, A.S., ed. Esquistossomose mansoni. São Paulo, Ed. USP, 1970. p. 13-30.

32. PARAENSE, W.L. Fauna planorbídica do Brasil. In: Lacaz, C. da S. et al. Introdução à geografia médica do Brasil. São Paulo, Ed. Edgar Blucher/Ed. USP, 1972. p. 213-39.

33. PARAENSE, W.L. The distribution of molluscan vectors of schistosomiasis in the Americas. Brasília méd., 1/2 (11): 114, 1975.

34. PARAENSE, W.L. A survey of planorbid molluses in the Amazonian region of Brazil. Mem. Inst. Oswaldo Cruz, Rio de Janeiro, 78: 343-61, 1983.

35. PARAENSE, W.L.; SOUZA, P.E.F.P. de; BRAUN, R.F. Novos focos de transmissão do Schistosoma mansoni no Estado do Pará. Mem. Inst. Oswaldo Cruz, Rio de Janeiro, 79 . 389-91, 1984.

36. PARAENSE, W.L. A xistosomose mansoni no Pará. In: Instituto Evandro Chagas: 50 anos de contribuição às ciências biológicas e à medicina tropical. Belém, Fundação Serviços de Saúde Pública, 1986, v. 1, p. 207-19.
37. PARAENSE, W.L. Control of Schistosomiasis mansoni: an outlook from currente expectation. Mem. Inst. Oswaldo Cruz, Rio de Janeiro, 82: 1-12, 1987.

38. REY, L. Parasitologia. Rio de Janeiro, Ed. Guanabara Koogan, 1973.

39. SUPERINTENDENCIA DE CAMPANHAS DE SAÚDE PÚBLICA (SUCAM), Planejamento de atividades operacionais em campanhas e controle de endemias na área de influência do Projeto Sobradinho. Brasília. 1974.

40. THOMAS, J.D.; GOLDSWORTHY, G.J.; BENJAMIN, M. Studies on the chemical ecology of Biomphalaria glabrata: the effects of chemical conditioning by the snails kept at various densities, on their growth and metabolism. J. zool., London, 175: 421-37, 1975.

Recebido para publicaçāo em 31/01/1991. Reapresentado em 04/06/1991. Aprovado para publicação em 14/06/1991. 